\title{
LA CIUDAD PERCIBIDA: CUESTIONES METODOLÓGICAS EN LA INVESTIGACIÓN DE AMBIENTES SONOROS URBANOS
}

\author{
Soledad Martínez Rodríguez ${ }^{1}$
}

\section{El cuerpo, paradigma de reflexión}

El estudio de la ciudad abre para los antropólogos una multiplicidad de campos de observación: la ciudad como imagen, como discurso, como espacio político, como espacio de consumo, modos de sociabilidad en la ciudad, etc. Este trabajo se enmarca dentro de la perspectiva del estudio de la experiencia urbana que se enfoca en las prácticas de los habitantes de la ciudad y más específicamente en la relación cuerpociudad.

Durante los últimos años hemos observado un creciente interés por la consideración del cuerpo en nuestras investigaciones. Los sujetos no son considerados sólo producto de sus procesos mentales o cognitivos, éstos están a su vez ligados a procesos sensoriales y perceptivos en los cuales la dimensión corporal es fundamental. Se ha comenzado a utilizar el concepto de embodiment (Csordas, 1994: 12; Ingold, 2000: 170) para hacer referencia no sólo a la incorporación de lo sensorial y corporal como un elemento más en el estudio de lo social, sino a su consideración como un elemento abordable en sí, asumiendo que existe un conocimiento que se produce desde la experiencia sensorial, desde las prácticas corporales, que existe -a fin de cuentas- un pensar con el cuerpo. Según María Luz Esteban en su artículo Antropología encarnada. Antropología desde una misma, aunque el término embodiment no sea utilizado en el mismo sentido todas las veces, se puede consensuar que a través de él se busca superar "la idea de que lo social se inscribe en el cuerpo, para hablar de lo corporal como auténtico campo de la cultura” (Esteban, 2004: 3). La traducción de este concepto es algo confusa para el castellano, sería algo así como “encarnación” o "incorporación”. Para evitar confusiones y para no perder la riqueza del término en inglés, se preferirá hablar de embodiment.

\footnotetext{
${ }^{1}$ Universidad de Barcelona, Espanha.
} 
En resumidas cuentas, podemos situar este giro o la incorporación de esta nueva perspectiva hacia lo corporal y sensitivo a fines de la década de los 70 y durante la década de los 80 , no sólo en antropología sino a nivel general dentro de las ciencias sociales. Hasta entonces el foco de atención estaba centrado en un paradigma simbólico e interpretativo. Siguiendo a Csordas, profesor de antropología de la Universidad de San Diego, dos de los autores decisivos para este cambio de perspectiva son Bourdieu y Merleau-Ponty. En ellos, de hecho, fundamenta su ensayo Embodiment as a Paradigm for Anthropology. El giro que proponen ambos tiene relación con superar el dualismo sujeto-objeto en función del cual la mente es concebida en contraposición al cuerpo:

Ambos intentan no mediar sino colapsar estas dualidades y el principio metodológico del embodiment es usado por ambos con tal fin. Para lograr el quiebre de las dualidades se requiere una figura metodológica del cuerpo no dual, o sea, no distinta de -o en interacción con- un principio mental opuesto. Así, para MerleauPonty el cuerpo es 'escenario en relación con el mundo' y la conciencia es el cuerpo proyectándose él mismo en el mundo; para Bourdieu el cuerpo socialmente formado es el 'principio generador y unificador de todas las prácticas' y la conciencia es una forma de cálculo estratégico fusionada con un sistema de potencialidades objetivas. (Csordas, 1990: 8) ${ }^{2}$

En ambas definiciones lo corporal no es ya sólo un objeto sobre el cual lo cultural y lo social se inscriben determinándolo, sino que pasa a ser sujeto agente de lo cultural y lo social.

Dentro del paradigma del embodiment se busca ir más allá de la representación del cuerpo. El cuerpo es considerado como base existencial de la cultura (Csordas, 1994) y por lo tanto, ya no interesa sólo por lo que se dice de él (ámbito de la representación), sino por lo que él mismo dice. Este giro que busca ir más allá de la representación (existen realidades abordables más allá de su propia representación) también se ha desarrollado en otras disciplinas como la geografía, algunos lo han denominado "non-representational theory", otros hablan de "more than representational". Hayden Lorimer, introduce uno de sus artículos sobre lo "more than representational" de la siguiente manera:

Primeramente, el fenómeno en cuestión parece considerable sólo por su insignificancia aparente. El foco apunta hacia cómo la vida toma forma y adquiere expresión en las experiencias compartidas, rutinas diarias, encuentros fugaces, movimientos corporales, provocaciones

\footnotetext{
${ }^{2}$ Traducción libre por parte de la autora de las citas a lo largo del artículo.
} 
precognitivas, habilidades prácticas, intensidades afectivas, estímulos constantes, interacciones ordinarias y disposiciones sensoriales. (Lorimer, 2005: 84)

Básicamente es una orientación hacia lo tangible, hacia lo inmediatamente dado, hacia el mundo de la vida, para utilizar el famoso concepto usado por la fenomenología, desarrollo teórico fundamental para el paradigma del embodiment (Csordas, 1990: 9; Ingold, 2000: 168-171). Uno de los campos que se abren al considerar el cuerpo del modo propuesto es justamente la investigación en torno a la percepción y la relación del cuerpo con su medio ambiente y la materialidad que lo rodea. Si se trata de hacer referencias, no podemos dejar de nombrar a Georg Simmel y sus estimulantes indagaciones sobre la vida moderna en general y en particular sobre lo urbano y el espacio público, donde dedicó líneas para pensar lo sensorial en la ciudad. En su Sociología de los sentidos realiza, en palabras de Isaac Joseph: "un análisis de las formas de sensibilidad concretas movilizadas por la experiencia urbana." (Joseph, 1988:52)

Lo que nos importa concretamente es el sujeto considerado en su dimensión sensorial -además de su dimensión cognitiva- en relación con su medio ambiente, en el caso particular del medio urbano. El habitante tiene una experiencia sensorial del medio urbano, lo escucha, lo siente, lo ve, lo huele en su andar cotidiano del día a día. Esta realidad perceptiva es constitutiva de la experiencia de la ciudad. Una dimensión fundamental del despliegue de lo social y de lo cultural en el medio urbano es el de sus características sensoriales y materiales. Especialmente porque lo urbano nos pone ante un escenario medio ambiental específico, con una sobreabundancia de elementos sensorialmente estimulantes:

En el ensayo 'La metrópolis y la vida mental' Simmel describe cómo la experiencia de la ciudad moderna es única en su sobreestimulación sensorial. La gran cantidad de estímulos sensoriales con los cuales el individuo es confrontado lleva a 'la intensificación de la vida emocional debido al rápido y continuo cambio de los estímulos externos e interno ${ }^{3}$. (Degen, 2008: 39)

Al mencionar el desarrollo del embodiment como paradigma, la intención ha sido fundamentar de manera puntual que dentro de las ciencias sociales existe una línea investigativa ya consolidada que nos lleva a reflexionar sobre este plano de lo material, de lo cotidiano, de las prácticas, de lo sensorial, de lo corporal en el amplio

${ }^{3}$ Citado por la autora de Simmel, G. "The Metropolis and Mental Life" en Levine, D.N. (ed.) Individuality and Social Forms: Selected Wrintings. 1971. Chicago: University of Chicago Press. 
sentido del término. Csordas nos dice al inicio de su artículo Embodiment as Paradigm for Anthropology:

Por paradigma me refiero simplemente a una perspectiva metodológica consistente que anima a un re-análisis de los datos existentes y sugiere nuevas preguntas para la investigación empírica. También debo señalar que el paradigma del embodiment trasciende diferentes metodologías (...) Esta aproximación al embodiment parte del postulado metodológico en el que el cuerpo no es un objeto para ser estudiado en relación a la cultura, en cambio es considerado como un ámbito propio, en otras palabras, como la base existencial de la cultura. (Csordas, T. 1990: 5)

Nos quedamos con la última frase: "como la base existencial de la cultura". Es justamente el marco de las investigaciones que se han comentado y de las aproximaciones metodológicas que revisaremos. Todo hecho cultural y social necesariamente tiene una lectura desde el cuerpo, oculta a nuestras reflexiones hasta hace poco, pero necesaria de explorar en cuanto puede iluminar nuevas preguntas y respuestas, tal como nos dice Csordas. Sea llamado embodiment, more than representational o non-representational, lo relevante es que ahora nuestras aproximaciones toman el cuerpo y lo sensorial como objeto de estudio con validez propia y no como un elemento anecdótico dentro de un contexto de investigación mayor.

Por eso es fundamental la perspectiva que nos ofrece el embodiment. El ámbito de lo sensible se perfila como un área de investigación que puede entregarnos claves no sólo para entender cómo el ser humano habita la ciudad, sino también para poder, a través de la consideración de lo sensible como un elemento central de la experiencia urbana, ayudar a la práctica efectiva de la construcción de los espacios públicos e intentar tender puentes que comuniquen la actividad de los sujetos planificadores de la ciudad con sus habitantes y/o usuarios. En este sentido y para cerrar este apartado, cito a dos investigadores franceses que tienen larga trayectoria en el estudio de la dimensión sensorial de los ambientes ${ }^{4}$ :

En efecto, nuestro cuerpo habita el espacio por medio de cada uno de sus sentidos (...) Antes que hablar de espacio público en el sentido abstracto y totalizante del término, parece más pertinente interrogar la diversidad de los espacios sensoriales. (Chelkoff y Thibaud, 1992: 7)

\footnotetext{
${ }^{4}$ Citado por Chelkoff, G. y Thibaud, J.P. de Augoyard, F. "Du lien social à entendre" Actes du XIIIe colloque de l'AISLF: Le lien social, Genève, Université de Genève.1989.
} 
El conjunto de métodos que se revisará además de estar enmarcado en el estudio de lo sensorial, lo está en el estudio del ambiente. La pregunta principal gira en torno al modo mediante el cual el sujeto se relaciona con esta otra materialidad más allá de las fronteras de su propia corporalidad: el paisaje, el entorno. Y más específicamente, el medio ambiente construido, el espacio de la ciudad.

\section{El sonido, condiciones de investigación}

La experiencia sensorial de la ciudad se desenvuelve en un estado general de desatención o inconsciencia, lo que dificulta su investigación. La escritora inglesa Virginia Woolf ha hecho alusión al estado de embotamiento que se experimenta debido a la abundancia de estímulos sensoriales simultáneos:

En la duración o en la brevedad, el paseante callejero es hipersensorialista. De ahí su frecuente sensación de embotamiento, de saciedad dulzona y sosa, de ahí su sonambulismo. De alguna manera es éste el precio que paga por su contrato con la calle. Sólo toma posesión de ella por la mirada ${ }^{5}$. (Joseph, 1988: 49)

Dentro de este embotamiento, en medio de la desatención y/o inconsciencia perceptiva, cada sentido no participa por igual. Podríamos hablar de la existencia de una jerarquía sensorial dentro de la cual la posición central es ocupada por la vista, tal como señalaba Woolf en la cita anterior "sólo toma posesión de ella por la mirada". Es tan sencillo como pedirle a alguien que describa una ciudad, probablemente las principales características corresponderán a apreciaciones visuales, los olores se nos confunden, los sonidos también, la textura a pesar de ser extremadamente determinante no la recordamos con facilidad. En su trabajo Sensing Cities, Mónica Degen, hace alusión a este predominio de lo visual en la construcción y percepción de las ciudades:

La visión ha sido tenida históricamente como el sentido más importante, y ha tendido a ser puesto en la cima de un 'orden sensorial'. En la jerarquía aristotélica de los sentidos, la visión y el oído fueron considerados como 'sentidos humanos', el tacto y el gusto fueron considerados como inferiores y animales (Synnott 1991). Aunque una definición así de estricta no es más el caso, o al menos no abiertamente explicitada, las afirmaciones basadas en observaciones visuales siguen predominando en el modo de investigar en ciencias. La mayoría de las veces el trabajo de campo aún se basa en ver lugares, con la consecuencia de que muchas de las

${ }^{5}$ Citado por Joseph, I. de "Au hasard des rues. Une aventure londonienne" en Le Mort de la Phalène. Seuil, 1968. p. 127-140. 
cualidades del lugar son reducidas sólo a lo visual (Igham et al. 1999; Porteous 1991). Las dimensiones no visuales de la experiencia suelen ser ignoradas o relegadas al mundo de la literatura, para evocar el 'carácter' o la 'atmósfera' de los lugares. (2008: 42)

Este orden jerárquico de los sentidos en la ciudad puede verse expresado tanto desde la vereda de los que planifican y diseñan la ciudad como desde la percepción y lectura de los propios usuarios. Probablemente la prevalencia de lo visual sobre el resto de los sentidos sea efectivamente una característica cultural general. Aunque en la alusión que se hace a Aristóteles se dice que tanto el ver como el oír son considerados como sentidos superiores es innegable la preponderancia de la vista. Hace poco estuve en una biblioteca en la cual el medio de acceso de una planta a otra eran escaleras mecánicas. Muchas veces el ambiente sonoro parecía más el de una fábrica que el de una biblioteca. ¿Es que nadie pensó en el ruido? Puede ser, no lo tenemos del todo instituido como una variable a la hora de diseñar espacios. Se imagina visualmente y no desde una integridad corporal. Los programas de diseño como el AutoCAD consisten básicamente en una simulación visual (dicho de manera simple), quedando oculto todo el resto de elementos sensoriales que participan en la configuración del espacio. (Sennett, 2009: 55-62)

Una de las consecuencias de la existencia de esta jerarquía sensorial es la escasez de herramientas tanto léxicas como cognitivas para distinguir y por lo tanto, recordar las otras percepciones sensoriales distintas de lo visual. Nuestro vocabulario no es tan amplio a la hora de hablar por ejemplo, de sonidos, que es el sentido que nos ocupa en este trabajo. La distinción es la base de la construcción lingüística, no podemos hablar de lo que no podemos diferenciar, y a su vez, el poder narrar las experiencias ayuda a que ellas pasen a incorporarse en nuestra memoria de un modo tal que luego podamos recurrir voluntariamente a ellas. No es extraño que los olores y los sonidos nos sean tan mágicamente evocadores, de manera sorpresiva muchas de las veces, y de un modo casi incontrolable. Un olor nos lleva a un lugar, pero es más difícil que en medio del proceso de descripción de aquel lugar recordemos necesariamente los aromas que hay en él. Probablemente incorporamos estos elementos de manera indiferenciada a una memoria corporal que no se narra, y esa falta de narración hace que luego, sea más difícil recordarlos y considerarlos como elementos del medio ambiente: "En la cultura actual, el fenómeno sonoro banal no es representable en cuanto a que no es comunicable socialmente. Su enunciación es estimada a priori insignificante". (Augoyard, 2001: 151) 
Esta dificultad para recordar elementos sensoriales auditivos u olfativos, puede responder también a que ambos sentidos tienen la particularidad de ser ubicuos en su radio de acción: se propagan fácilmente. Es difícil aislar el sonido o aislar los olores. Podríamos pensar que esta característica expansiva los hace difícil de ubicar y se quedan dispersos materialmente (nos es difícil ubicar su fuente de origen) y dispersos en la memoria: "Con el sentido del olfato y del oído estamos expuestos a una estimulación externa a la que no nos podemos resistir. Olores y sonidos, aunque producidos por un objeto, escapan de él y no son más prisioneros de formas cerradas". (Degen, M. 2008:44)

Ante este escenario de embotamiento y jerarquía sensorial cabría preguntarnos si será posible el estudio de la percepción auditiva del espacio urbano. ¿Cómo narrar lo que no tiene status de memorable? Dentro de una jerarquía sensitiva que privilegia lo visual, el sonido, junto con el resto de los sentidos quedan en paréntesis. ¿Qué sonidos escuchas en el trayecto a casa? Pregunta de no inmediata respuesta.

\section{Revisión de métodos}

Como hemos dicho, las percepciones auditivas cotidianas del medio urbano quedan en el borde de lo distinguible, de lo recordable, y por lo tanto, de lo narrable. Este hecho nos pone ante un desafío metodológico dado que nuestras metodologías clásicas de investigación se apoyan ampliamente en lo que se cuenta, en preguntas y respuestas, en conversaciones y entrevistas -en la representación-. Uno de nuestros principales recursos es la palabra y la memoria de las personas.

Esta forma de investigación se vuelve limitada a la hora de estudiar la dimensión sensorial del espacio, en este caso, del espacio urbano caracterizado, como señalamos, por una superabundancia de estímulos sensoriales. ¿Cómo podemos sacar del estado casi inconsciente este tipo de experiencias sensoriales de la ciudad? ¿Cómo podemos propiciar una narración de lo sensorial? Requerimos diseñar herramientas metodológicas que apunten a la experiencia a la vez que al discurso, dice François Augoyard:

"La unidad de una situación sonora concreta se realiza en la vivencia de los auditores habitando el lugar observado. Dicho de otra manera, ni las medidas físicas, ni el registro, ni las descripciones, ni las observaciones etnográficas son suficientes. Sólo las expresiones de los actores del espacio urbano, expertos o no, pueden expresar el sentido preciso y así, la unidad de una situación sonora". (2001: 129) 
No basta sólo saber lo que se piensa y se recuerda a partir de las palabras (ya vimos sus limitaciones), sino que hace falta acceder al espacio mismo en el que la percepción se genera, ya sea en tiempo presente o trabajando con la reactivación de la experiencia, pero con ciertas claves que nos permitan llegar a los espacios de la memoria que están más alejados de las distinciones narrativas.

Ya planteado el problema que entraña el sonido en cuanto a su investigación como elemento del medio ambiente: su naturaleza expansiva y su dificultad para ser distinguido y recordado, pasaremos a hacer revisión de algunos de los métodos propuestos por investigadores franceses agrupados en torno al Centro de investigación sobre el espacio sonoro y el entorno urbano (CRESSON) que se dedica al estudio de estas temáticas con un grupo interdisciplinar. Los métodos que se describen a continuación están reunidos en una misma obra que se titula: L'espace urbain en méthodes. La revisión no pretende reproducir todas las indicaciones y recomendaciones que los autores hacen para la aplicación efectiva de los métodos, más bien pretende ser una ventana para pensar este tipo de aproximaciones.

\section{Entrevista sobre escucha reactivada}

Para comprender este método es necesario comprender la distinción que Augoyard propone entre el "sonido propiamente tal" (caracterizado físicamente y reproducible casi de forma exacta) y el "sonido vivido" (inevitablemente interpretado, deformado). Esta distinción es fundamental para proponer una investigación sobre el sonido. Winkler también llega a una distinción similar cuando habla de "bucear en el mundo sonoro de la vida" ${ }^{6}(2001: 33)$. Al usar el término "bucear" Winkler busca resaltar la idea de un ambiente sonoro que nos rodea completamente llenando tanto el tiempo como el espacio, al modo de un océano en el que estamos inmersos. En medio de este océano sonoro, existe un ambiente sonoro y un ambiente sonoro "escuchado", es decir que leemos e interpretamos, lo que se corresponde con la distinción realizada por Augoyard. Existiría un "mundo sonoro" y múltiples "mundos escuchados" (Winkler, 2001: 35). Lo que nos interesa a la hora de investigar las sonoridades de los espacios urbanos es justamente acceder a esa interpretación, al "sonido vivido", el cual estará siempre relacionado de manera determinante por un contexto, por una

\footnotetext{
${ }^{6}$ La frase original es: "Diving into the sonic lifeworld". El término lifeworld corresponde al usado por la fenomenología, derivado del alemán lebenswelt y traducido al castellano generalmente como "mundo de la vida".
} 
"situación sonora" (Augoyard, 2001: 129) la que se definirá por el conjunto de “sonidos propiamente tales" y "sonidos vividos".

Este método de investigación trata de "recoger las reacciones de los habitantes y de los usuarios a quiénes se les hace escuchar los sonidos de su propio entorno" (Augoyard, 2001: 130). Permite por una parte distanciar a los sujetos de su ambiente sonoro familiar favoreciendo la creación de una representación del espacio sonoro cotidiano y por otra parte, reencontrar lo vivido, crear un canal donde el sujeto pueda volver a entrar en sus experiencias sensibles ya vividas, teniendo en cuenta que sin duda, algunos de los sonidos serán por primera vez percibidos en el momento de la reescucha en medio de la entrevista.

Augoyard establece una gran cantidad de posibilidades y especificaciones para la aplicación del método. Dado el marco de este artículo, que pretende una reflexión acerca del modo en el que podemos afrontar las investigaciones de los ambientes sensibles -sonoros- cotidianos antes vividos que reflexionados o recordados, solo haré un resumen general del método en cuanto nos permite acceder a ese espacio de la experiencia más allá del lenguaje y la representación.

La experiencia en su aplicación lleva a Augoyard a la distinción entre investigaciones de dos tipos. Tipo A: investigación de la vida sonora de un lugar. De tipo B: investigaciones que buscan corroborar hipótesis acerca de las percepciones, representaciones y prácticas sonoras en general. Debido al marco de nuestro trabajo, nos centraremos en las investigaciones de tipo A.

Primeramente se realiza una investigación preparatoria para obtener el material sonoro que será luego dado a escuchar: la idea es grabar el máximo de material sonoro posible para luego seleccionar lo más pertinente según lo objetivos que se persiguen. Se trabaja después en el montaje de secuencias sonoras ${ }^{7}$ cuyo límite será el propio cansancio del entrevistado (máximo una hora y media). En esta etapa preparatoria es necesario tener una experiencia próxima e intensiva del lugar que implica realizar entrevistas libres en el sitio, que tendrán el doble objeto de sostener conversaciones acerca de las características sensibles del lugar y además proponer participar a los habitantes o usuarios en la investigación (esto será más fácil en lugares residenciales que en el espacio público donde nos encontraremos con las

\footnotetext{
${ }^{7}$ Augoyard recomienda ciertos criterios a tener en cuenta al momento de elegir los sonidos que luego conformarán las secuencias a ser escuchadas, lo que sería muy largo de resumir aquí.
} 
reservas del anonimato y las dificultades del continuo tránsito de los usuarios del lugar). En esta etapa se recomienda complementar con la mayor cantidad de datos: croquis, topología del lugar, etc.

Este método puede ser aplicado tanto en entrevistas individuales como grupales, teniendo cada modalidad sus ventajas y desventajas. Las entrevistas personales no presentan inconvenientes, sólo hay que estar atento a las apreciaciones del entrevistado que pueden ser profusas para ciertas secuencias y menores para otras. Las entrevistas grupales poseen una riqueza mayor, según Augoyard, debido a la interacción de los participantes quiénes se corrigen, se preguntan mutuamente, se contradicen y negocian interpretaciones. El cuidado hay que tenerlo con las dinámicas interpersonales de grupo que puedan generarse (si hay conocidos, si uno toma la voz, si otros se retrotraen). El lugar debe ser adecuadamente elegido, puede ser un espacio en el mismo lugar estudiado o en un lugar neutro. La escucha es flexible, puede volverse atrás (por este motivo es necesaria la construcción de un guión lo más detallado posible de las secuencias y de sus marcas temporales para poder volver a ellas sin perder tiempo en buscar), las personas pueden comentar mientras escuchan. El investigador tendrá que administrar estas flexibilidades, por ejemplo una escucha demasiado interrumpida puede convertirse en un ejercicio de reconocimiento que no nos lleve a esferas más profundas de la experiencia sonora.

A rasgos generales, la escucha reactivada apunta a "entrar con los entrevistados en una reminiscencia sonora y encontrar junto a ellos las voces singulares de la escucha in situ" (Augoyard, 2001: 150). En este sentido se reitera en el texto la idea de que este método se basa en una escucha en común que involucra tanto al investigador como al entrevistado:

La escucha reactivada no se conjuga solamente en primera persona; es, de hecho, una escucha compartida. Sabemos que no es fácil hacer emerger de manera significativa fenómenos a la vez omnipresentes y raramente conscientes, tal es el caso de los sonidos ordinarios. Esta sordera psicológica afecta tanto al investigador como al sujeto de la investigación (...) Al investigador le hace falta la experiencia integral del usuario del lugar, al habitante, el conocimiento experto y la aptitud de ser consciente del ambiente sonoro". (Augoyard 2001: 134)

En la escucha reactivada la secuencia sonora es el enganche principal, en ese sentido rompe la relación tradicional cara a cara entre el entrevistado y el entrevistador y las interferencias que en esa interacción puedan generarse, llevando al 
entrevistado mediante el material sensible (secuencias sonoras) a proyectar su propia experiencia cotidiana. El entrevistado revive la experiencia sonora, por lo general olvidada o simplemente no percibida, y nos otorga la posibilidad de observarlo e interactuar con él dispuesto ante una parte de la experiencia misma. Esta característica lo posiciona como un método que intenta resolver las características del sonido en la ciudad, su carácter inconsciente habitual, y efectivamente nos ayuda a acercarnos a la experiencia misma de manera tal de poder darle, en conjunto con el entrevistado, una posibilidad de expresión.

\section{Observación Recurrente - Recorridos Comentados}

La observación recurrente nace inspirada en la entrevista sobre escucha reactivada. Se propone el trabajo de manera indirecta, interpretativa y acumulativa. Si bien no es un método pensado para trabajar con ambientes sonoros específicamente, puede ser interesante su aplicación en cuanto a que permite entrar en contacto no sólo con la experiencia aislada del sonido sino que también con la globalidad de la situación (teniendo en cuenta que sentidos como el olfato o el tacto, quedarán siempre en este tipo de técnicas de reactivación, relegados).

Básicamente se trata de la utilización de documentos fotográficos o videográficos de situaciones urbanas específicas para presentarlas ante un grupo de especialistas o habitantes del lugar y recoger sus comentarios e interpretaciones. Para el caso del sonido quizás pueda ser más útil recurrir a documentos videográficos. De todas formas, la no sonoridad de los documentos fotográficos podría ser utilizada como un modo de "evocación por ausencia": ¿qué sonidos asociarían a la imagen de un lugar determinado? Las posibilidades son amplias.

El punto central de este método, según Pascal Amphoux, es "hacer hablar a la ciudad". No sólo hablar de la ciudad que es lo que podría darse en una observación etnográfica clásica, sino que a través de la representación del video lograr una observación retrospectiva controlable y repetible lo que nos abre la posibilidad de múltiples miradas sobre el ambiente en cuestión.

En la práctica, el investigador debe trabajar en conjunto con un realizador. Se deben elegir situaciones o espacios que permitan abordar de mejor manera el objeto de estudio con la ayuda de consultas previas a los habitantes o usuarios del lugar. En otras palabras, que sea expresivo de lo que sea desea captar. En la realización del video o de la secuencia fotográfica, deben ser considerados ciertos elementos como 
localización, intención (contenido, concepto dominante del ambiente, calificativos), composición (elementos formales: planos generales, travellings, sonido, efectos especiales, etc.) e información (horarios, días favorables para la realización, etc.). Es necesario ser conscientes de la existencia de un sesgo inevitable que será consecuencia de aquello que mostremos o dejemos de mostrar.

Luego de la realización del documento, cuya duración debería variar entre 1 y 2 minutos, la aplicación prosigue enseñando el material a especialistas de diferentes disciplinas y solicitándoles que describan el contenido y que señalen elementos de interpretación. Estas observaciones podrán ayudar a precisar el contenido de los documentos. La idea es sobrepasar la subjetividad inicial e intentar objetivizar por medio de la recurrencia, las características de los lugares o situaciones observadas. Finalmente se hace la entrevista con los habitantes o usuarios. Para lograr cierta objetividad debería llegarse a que por cada video participaran entre 5 y 10 personas. De todos modos la muestra no será "representativa" en el estricto sentido del término, Amphoux dice que podríamos decir que es más bien "expresiva", y es justamente el máximo de expresividad lo que se busca con la aplicación de este método: "el principio mismo de la objetivación por recurrencia se basa, en efecto, en la diversidad a priori de las miradas fijadas en el videograma" (Amphoux, 2001: 158).

La duración de una entrevista satisfactoria irá desde la hora y media hasta las tres horas. Amphoux resume el guión de la entrevista de la siguiente manera:

a. recordar brevemente los objetivos de la investigación y los estereotipos de los cuales se quiere escapar.

b. ver una vez la secuencia y pedir a los participantes que comenten, la idea es ir de lo fáctico a lo interpretativo.

c. provocar la continuidad de la palabra de los participantes, revisando nuevamente el material algunas veces, parando si es requerido, volviendo a exponer las hipótesis, etc.

El último método, recorridos comentados, quizás sea el más conocido entre los que hemos revisado, y probablemente surge casi espontáneamente en el transcurso de algunas investigaciones. A diferencia de los dos anteriores, nos permite trabajar en un tiempo presente, es decir, estar en el momento de la percepción del ambiente.

El objetivo de este método es obtener las descripciones ordinarias de los transeúntes, sus percepciones en movimiento. Parte también con una observación previa del sitio. Luego, al realizar el recorrido con la personas, se les pide que hagan 
el ejercicio de describir los lugares. Se puede incorporar la perspectiva sensorial -que es la que nos interesa- y llevarlos a describir los lugares, por ejemplo, poniendo atención en las sonoridades. La persona tiene la libertad de retroceder, cambiar de vía, elegir algunos tramos de la ruta. Debido al esfuerzo que se moviliza, tanto de parte del investigador como del participante, se recomienda no sobrepasar los 20 minutos.

En un segundo momento el trayecto recorrido es reconstruido en conjunto con el habitante con la ayuda de un mapa si fuese muy complejo, o preferentemente, sin documento gráfico para llegar a observar cómo ha sido memorizado el recorrido. La idea es repetir un mismo trayecto con alrededor de 20 habitantes, intentando conseguir una muestra lo más variada posible. Se realiza un registro auditivo del recorrido (podría indagarse en la variante de un registro audiovisual) para luego poder volver a él y analizar los comentarios.

Este es una método simple en su descripción, porque responde a una situación común que es elevada al ámbito de la investigación: caminar acompañado. La forma recién descrita es la que aconseja J.P. Thibaud, sin embargo puede modificarse según las necesidades de profundidad, objetividad/subjetividad requeridas por cada investigación particular.

\section{Cierre}

A partir de las nuevas perspectivas de investigación que se nos presentan, lo que se refleja en la aparición del embodiment como paradigma, se pueden derivar estudios específicos tales como el de la dimensión sensible de las ciudades y específicamente de los ambientes sonoros urbanos. Se nos hace necesario encontrar y experimentar con la metodología de manera tal que nos permita abordar la experiencia sensorial en la ciudad. Como planteamos en un principio, una de las principales dificultades que debemos superar es el carácter no narrado de muchas de las percepciones cotidianas del medio urbano. Es justamente eso lo que une estos tres métodos, ellos buscan permitir la observación del investigador sobre la experiencia sensorial misma. La narración de la experiencia sensorial se propicia mediante la proyección y la descripción. Para comprender mejor esto se hace necesario pensar en función de dos pares conceptuales clave: recreación-proyección/acceso-descripción.

El primer par recreación-proyección corresponde a los métodos de entrevista sobre escucha reactivada y observación recurrente. En ambos casos el sujeto es puesto ante el estímulo sonoro que constituye parte de la experiencia que se desea 
investigar. Se recrea el acto de la percepción de manera dirigida en un espacio diferente al contexto original. Eso ayuda al sujeto a concebir y reflexionar sobre la experiencia específica como un todo, pues la escucha desencadenará mecanismos de reactivación de la memoria y la creación de imágenes. La secuencia sonora, fotográfica o videográfica escogida para la entrevista actúa como un primer estímulo que trae a la memoria experiencias quizás hasta entonces nunca narradas, lo que genera una proyección de la experiencia por parte del sujeto. Se habla de proyección justamente por la distancia que hay con el contexto original y con el espacio-tiempo de la experiencia en sí. Se conjugan elementos presentes (los de la secuencia elegida), elementos ausentes (los evocados, el contexto) y probablemente elementos inventados que aparecerán para intentar dar coherencia al relato (el sujeto proyecta una experiencia como un todo).

El segundo par acceso-descripción alude al método de recorridos acompañados, en él, a diferencia de los anteriores, se participa y observa en el espacio-tiempo mismo de la percepción. Por lo tanto podemos decir que de cierta manera, teniendo en cuenta la distorsión que siempre significa la presencia de un observador sobre las prácticas de un sujeto, tenemos acceso a la experiencia misma. Al estar compartiendo el contexto de la escucha, al estar percibiendo el conjunto, el tipo de datos que se obtenga será de carácter más descriptivo. El hecho del sujeto describiendo su propia experiencia nos pone en una situación privilegiada en la que podremos profundizar y a través de nuestras preguntas generar un vehículo de expresión de la experiencia.

Sin duda que lo proyectivo y lo descriptivo emergen en todos los casos de manera combinada y como un todo en la expresión del habitante. Aún así, creemos que por las características de cada situación se privilegia una más que otra, lo que nos ayuda a entender el carácter de cada método aludido. De todos modos, la expresión es la llave de entrada que nos permite trabajar con la experiencia sensorial urbana. Ya sea que tome la forma de descripción o proyección. Augoyard habla de "expresión habitante" (Augoyard, 1979: 21) y Amphoux, para el caso de la observación recurrente, de "muestra expresiva" (Amphoux, 2001:158). Antes que buscar la sola representación de la experiencia sonora en la ciudad, lo que nos llevaría a relegar nuevamente el cuerpo en nuestras investigaciones que es justamente lo que hemos criticado al hablar del paradigma del embodiment, se propicia el cuerpo como medio de expresión. Estos métodos persiguen abrir un espacio donde tanto cuerpo y voz 
puedan encontrar una vía de emerger desde lo indistinguible y entrar en un diálogo con el investigador, y no sólo con él, sino con el mismo sujeto que en este proceso ve aparecer experiencias que quizás no había reflexionado anteriormente.

A partir de revisiones de este tipo de metodologías que nos proponen una manera creativa de abordar el trabajo de campo usando los elementos tradicionales de manera no convencional, podemos proseguir un camino de innovación metodológica que nos lleve cada vez más cerca de esta dimensión cotidiana, efímera, ordinaria, que es la experiencia sensorial del medio ambiente.

\section{Referencias}

AMPHOUX, P. L'observation récurrente en L'espace urbain en méthodes. Marseille: Éditions Parenthèses, 2001. pp. 127-152.

AUGOYARD, J.F. L'entretien sur écoute réactivée en $L$ 'espace urbain en méthodes. Marseille: Éditions Parenthèses, 2001. pp. 127-152.

AUGOYARD, J.F. Pas à pas. Essai sur le cheminement quotidien en milieu urbain. Paris: Seuil, 1979.

CHELKOFF, G. y THIBAUD, J.P. L'espace public, modes sensibles: le regard sur la ville en Les Annales de la recherche urbaine. Espaces Publics [online] $\mathrm{n}^{\circ}$ 57-58; décembre 1992- mars 1993. pp. 7-16 [fecha de consulta: 20 mayo 2009]. Disponible en: http://halshs.archives-ouvertes.fr/docs/00/11/26/53/PDF/1992-JP-T-GC-ARTAnnales-espace-pdf

CSORDAS, T. Embodiment as a Paradigm for Anthropology en Ethos [online] vol.18, nº1. Marzo 1990. pp. 5-47 [fecha de consulta: 5 junio 2009] Disponible en: http://www.jstor.org/stable/pdfplus/640395.pdf

Embodiment and experience. The existential ground of culture and self.

Cambridge: Cambridge University Press, 1994.

DEGEN, M. Sensing Cities. London: Routledge, 2008.

ESTEBAN, M.L. Antropología encarnada. Antropología desde una misma en Papeles del CEIC (Centro de Estudios sobre la Identidad Colectiva), Universidad País Vasco. [online] 2004. [fecha de consulta: 5 junio 2009] Disponible en: http://www.ehu.es/CEIC/papeles/12.pdf

INGOLD, T. The Perception of the Environment. Essays in livelihood, dwelling and skill. London: Routledge, 2000.

JOSEPH, I. El transeúnte y el espacio urbano. Buenos Aires: Gedisa, 1988.

LORIMER, H. Cultural geography: the busyness of being „more-thanrepresentational" en Progress in Human Geography [online] 2005; 29, 1 [fecha de consulta: 24 enero 2008] Disponible en: http://phg.sagepub.com/cgi/content/refs/29/1/83

SENNETT, R. El artesano. Barcelona: Anagrama, 2009.

THIBAUD, J.P. La méthode des parcours commentés en L'espace urbain en méthodes. Marseille: Éditions Parenthèses, 2001. pp. 127-152. 
